\title{
Safety Guidelines for PDT of Cutaneous Lesions
}

\author{
Yoshinari Matsumoto, Yoichi Akita \\ Department of Dermatology, Aichi Medical University, School of Medicine
}

\section{Purpose}

The purpose of these safety guidelines is to list the requirements and conditions that need to be strictly adhered to, in order to promote and ensure the safety of patients, doctors and medical personnel during a photodynamic therapy (PDT) procedure for dermatological, cutaneous lesions. In the field of dermatology, the most common type of PDT procedure is such where a topical photosensitizer, 5-aminolevulic acid (5ALA, ALA ), a porphyrin precursor, has been applied to the skin which is subsequently irradiated with laser energy of a specific wavelength. These guidelines will focus on PDT with ALA (ALA-PDT) ${ }^{1-6}$ for cutaneous lesions.

\section{Qualification and requirements of the medical facility where ALA-PDT is to be performed}

In order that ALA-PDT can be performed for dermatological cutaneous lesions, the medical facility concerned must possess adequate hardware and equipment including laser or light devices delivering light energy at an appropriate wavelength along with medical personnel who are well trained and capable of operating the hardware and devices and who have a thorough and specialized knowledge of dermatology and also a thorough knowledge of the photosensitizer being used for the PDT procedure.

Such a medical facility shall be a facility accredited by the Japanese Dermatological Association as an instructional or certified facility.

Addressee for Correspondence:

Yoshinari Matsumoto

1-1 Yazakokarimata, Nagakute,

Aichi, 480-1195, Japan

TEL:+81-561-62-3311 / FAX:+81-561-63-9914

E-mail: ymatsu@aichi-med-u.ac.jp

\section{Adherence to the user's manuals and appendices}

Doctors and medical personnel who are performing PDT must have thoroughly read and comprehended the attached documents and instructions for both the photosensitizer, ALA, and the laser or light devices which are to be used for activation during the PDT procedure. The medical facility must keep these documents and instructions at the site of the PDT procedure and have them available at all times.

The head of the medical facility is required to ensure that the facility's Laser Safety Officer (LSO) adheres to the content of the instructions regarding the specific device and its environment while the LSO is required to ensure that the users of the laser device adhere to the content of the instructions for its safe and effective use.

If and when a complication due to medication or malfunction of the laser device occurs, a report on the nature of the complication and or malfunction must immediately be sent to the pharmaceutical company, laser manufacturer and, if warranted, to the appropriate regulatory authorities.

\section{Cutaneous lesions indicated for ALA-PDT 1-13}

In the field of dermatology, PDT using ALA as the photosensitizer is the most common method. Typical cutaneous lesions indicated for PDT are superficial skin malignancies such as solar keratoses, Bowen's disease and superficial type basal cell carcinomas. However non-malignant lesions such as acne vulgaris, alopecia areata, sebaceous hyperplasia, refractory viral warts, skin sarcoidosis, psoriasis vulgaris, scleroderma, and bacterial or fungal infections of the skin may also be treated with ALA-PDT. ALA-PDT may also be used for enhancement of wound healing or for photorejuvenation. 


\section{Light sources and indications for their use}

The wavelengths used for ALA-PDT in the field of dermatology are the wavelengths at the absorption peaks of specific porphyrins, namely protoporphyrin IX (PpIX) and to a lesser extent, coproporphyrin III (CpIII), heme cycle-associated derivatives of ALA. Both these porphyrins have absorption peaks at the wavelengths of $415 \mathrm{~nm}$ (Soret band), and at $510 \mathrm{~nm}, 545$ $\mathrm{nm}, 580 \mathrm{~nm}$ and $630 \mathrm{~nm}$ (Q-band). Due to the comparatively poor penetration of light energy into living human skin at the shorter visible wavelengths due to absorption in the competing biological chromophores of melanin and blood, in general the red light at or around $630 \mathrm{~nm}$ is used for its deeper skin penetration. The use of laser energy is advocated as the light source but light from filtered flash lamp sources and new-generation LED systems has shown comparable results. Although the absorption coefficient of these porphyrins is greatest around the wavelength of $415 \mathrm{~nm}$, skin penetration of light at this wavelength is low and selection of this wavelength for activation of ALA requires caution in choosing appropriate lesions, such as limiting the use of this wavelength to superficial lesions less than $1 \mathrm{~mm}$ in depth e.g., actinic keratoses.

\section{Management of the Patient}

The doctor must be aware of the patient's medical history and background and examine all aspects of the patient that may affect or be affected by the PDT procedure. If the lesion is located in the torso or limbs the patient must be protected by wearing protective goggles, and if the lesion is located in the neck or head region, the patient must be blindfolded with a lightproof, incombustible fabric. If the lesion is located near the eyes, the patient must be protected with sterile ocular contact shells using ophthalmic analgesia.

\section{Contraindications of ALA-PDT in the field of dermatology}

ALA-PDT in the field of dermatology is contraindicated for those with past histories of hyperphotosensitivity, those with porphyria and those who are allergic or have shown hypersensitivity to ALA.

\section{Points where special attention should be paid for a safe PDT procedure}

Points where special attention should be paid for a safe ALA-PDT procedure are listed below in chronological order of the treatment.

\section{1) Pre-treatment examination}

Confirm that the lesion is indicated for the ALAPDT procedure by close and thorough evaluation of skin biopsy specimen.

\section{2) Management of the treatment room prior} to the ALA-PDT

During the ALA-PDT procedure, attention to light including reflected and scattered light must be paid. The environment should be as such that no light may escape and that the room has sufficient number of protective goggles for all who are required to enter the room. If there are multiple light sources in the room, protective goggles for all respective wavelengths are required.

\section{3) Inspection of the equipment prior to the ALA-PDT procedure}

The laser hardware or other light source for ALA activation must be both visually and functionally inspected prior to the application of the photosensitizer. The output power of the light source must be checked with a power meter to confirm that the correct irradiance is being delivered.

\section{4) Preparation of the lesion prior to the}

\section{ALA-PDT procedure}

Hyperkeratosis of the lesion may diminish the permeability of the skin to ALA, and a high melanin content of the stratum granulosum and stratum spinosum will offer a competing chromophore to the incident light energy, particularly at the shorter visible light wavelengths, and decrease the penetration depth of the light, hence hindering the effect of the PDT procedure.

Therefore exfoliation of the stratum corneum should be performed by application of ointments such as zinc oxide or urea. If the stratum corneum cannot be removed or medication alone is insufficient, consider tape stripping or the use of instantaneous adhesives.

\section{5) Preparation and administration of ALA}

Dissolve ALA in hydrophilic ointment to make a 20\% (w/w) ALA preparation which should then be applied to the lesion $\left(50 \sim 200 \mathrm{mg} / \mathrm{cm}^{2}\right)$. The area to which the ALA has been applied should be covered with an occlusive dressing in order to increase the penetration of the ALA, and wrapped in aluminum foil to avoid light. Laser or light irradiation should start 4 to 6 hours after the application of the 
ALA ointment. In the case of ALA-PDT for acne vulgaris or facial photorejuvenation, the concentration of ALA should be reduced to avoid undue skin irritation. Ingestion of ALA may lead to severe hepatic dysfunction and hyperphotosensitivity, and perioral topical application of ALA should be performed with the utmost care and observation.

\section{6) Precautions during the ALA-PDT proce-} dure

(1) Considerations during irradiation of the target with light energy

During the ALA-PDT procedure, the patient, doctors and medical personnel are required to wear protective goggles, and the laser or light source must be used in accordance with the manufacturer's instructions and any other instructions from the LSO. Since there is almost no damage to normal tissue, make sure that the light irradiates the entire lesion by adjusting the irradiated area to include a few $\mathrm{mm}$ of the tissue beyond the periphery of the ALA-treated lesion margins. The most commonly indicated and observed PDT parameters are as follows. ${ }^{10,13}$

(Per single irradiation, but note that these parameters are not applicable to ALA-PDT for acne vulgaris or photorejuvenation)

- Energy density:

$$
50 \sim 100 \mathrm{~J} / \mathrm{cm}^{2}
$$

- Irradiance: $50 \sim 100 \mathrm{~mW} / \mathrm{cm}^{2}$

- Number of irradiations: $\quad 3 \sim 6$ sessions

- Interval between sessions: 1 week

(2) If the patient cannot withstand the pain during the PDT photoactivation stage, the session could be aborted or a coolant (ice water) may be applied to the patient to alleviate the pain followed with the continuation of the session; local anesthetics may be used (be careful of allergies or shock caused by local anesthetics); alternatively a forced air cooling system may be used.

(3) If and when any malfunction of the hardware and equipment occurs during the PDT procedure, such as abnormal laser output, temporarily suspend the session and check the machinery. If the problem cannot be solved, abort the session and reschedule to another date.

\section{7) After the ALA-PDT procedure}

After the ALA-PDT procedure, the area to which ALA was applied must be shielded from light for 2 days after the treatment, but otherwise the patient may resume their normal daily activities. Erythema and edema of the treated area may be seen in certain instances but will subside along with the pain within a few days and crusting will become evident. The average period for the healing would be 1 week, in case of a facial lesion. The treated area should be washed with warm water to wash off the remaining ALA if the pain is not too strong. Petrolatum jelly or ointments containing antibiotics should be applied daily to protect the treated area. Post-inflammatory hyperpigmentation will resolve within a few months in most cases but remain in a few. After confirmation of complete epithelialization of the area, prescription of topical hydroquinone ointments, oral tranexamic acid, or ascorbic acid should be considered. After the treatment is completed confirm that the treatment was effective. Periodic examinations and follow ups are warranted.

\section{8) Informed Consent}

PDT for cutaneous lesions is not covered by the Japanese National Health Insurance. Patients should be fully informed of the effect that can be anticipated by the treatment, the number, period and interval of treatments, complications and other treatment options. After patients have received such grounding regarding the treatment procedure, they must sign a written form of consent to undergo the procedure.

\section{Rules and stipulations for the manufac- turers and distributors of PDT related drugs and laser hardware}

1) Obligation of the manufacturers and distributors to offer complete information regarding the products through user's manual and appendices

All manufacturers and distributors of drugs and laser or other hardware associated with PDT must provide ample and sufficient information to institutions, medical and paramedical personnel through the user's manual and appendices, for the proper and safe usage of the drugs and laser/light devices. This may be done through technical seminars showing actual video footage of PDT procedures, either independently, or at meetings and congresses of relevant medical societies with the cooperation of those societies. The manufacturers and their distributors are required to disseminate information for the safe and effective treatment with PDT.

The content and information that are required in 
the user's manual are dictated in the "Instructions for the use of laser devices", release no. 524 Notice from the Division Head of the Evaluation and Licensing Division, Pharmaceutical Affairs Bureau of the Japanese Ministry of Health and Welfare, dated April 22nd, 1980. ${ }^{14}$

The content concerning checking and maintenance of laser devices must include:

(a) Daily pre-operative checks involving both visual and operational checks

(b) Intra-procedural checks (checking that the system is performing correctly while it is actually being used)

(c) Post-operative checks (to be performed at the end of the day, including checks to be performed on the day after the procedure, and cleaning up)

\section{2) Written confirmation of delivery of the laser/light devices}

Upon the delivery of the laser/light device, the distributor and the medical facility must sign and seal a written confirmation concerning the items listed below, abiding by Appendix 2 of "Rules and Regulations of the Manufacturer and Distributor" from the business communications of the Division of Medical Device Development, Pharmaceutical Affairs Bureau of the Japanese Ministry of Health Labour and Welfare, dated August 6th, 1991. 15 Two copies of this written confirmation must be made, each party keeping a single copy.

\section{【References】}

1: Kennedy JC, Pottier RH, Pross DC: Photodynamic therapy with endogenous protoporphyrin IX: basic principles and present clinical experiences. J Photochem Photobiol B, 6:143-148, 1990.

2: Pass HI: Photodynamic therapy in oncology: Mechanism and clinical use. J Natl Cancer Inst, 85:443-456, 1993.

3: Peng Q, Warloe T, Berg K et al: 5-aminolevulic acid-based photodynamic therapy. Clinical research and future challenges. Cancer, 79: 22822308, 1997.

4: Dougherty TJ, Gomer CJ, Henderson BW et al: Photodynamic therapy. J Natl Cancer Inst, 90: 889905, 1998

5: Matsumoto Y: Application of photodynamic therapy and diagnosis for dermatological lesions. Hifubyoushinryou, 22:235-239, 2000.
Subjects requiring confirmation upon delivery of the laser device

(1): That laser safety officers (LSOs, chief and deputy - at least 2 people) are assigned and present.

(2): That a registered users' list has been made

(3): That the planned users of the device are surgeons who are board certified by the Japanese Dermatological Association and the Japan Society for Laser Surgery and Medicine, and that the facility manager has the right to appoint the user of the device.

(4): That the user is technically qualified and has attended courses for handling the drug and laser/light device, laser safety management, and risk and danger prevention.

(5): That the laser/light device is key controlled, and that the safe keeping of the key has been determined.

(6): That appropriate protective goggles for the wavelength of the laser/light device are supplied.

(7): That a dedicated protective earth terminal is made available and the laser/light device is correctly connected to it.

\section{Amendments and changes to the guide- lines}

Changes and amendments to the guidelines will be made based on future evidence.

6: Itoh Y, Ishibashi A: Application of photodynamic therapy for dermatological lesions.

Hifubyoushinryou, 22:1185-1190, 2000.

7: Itoh Y, Ninomiya Y, Henta et al : Topical $\delta$ aminolevulic acid-based photodynamic therapy for Japanese actinic keratoses. J Dermatol, 27: 513-518, 2000.

8: Kalka K, Merk H, Mukhtar H: Photodynamic therapy in dermatology. J Am Acad Dermatol, 42: 389416, 2000.

9: Matsumoto Y: Principles and applications of photodynamic therapy. Hifu-sikkann no saisin-irryou, sentan-irryou gijyutu kenkyujyo:5-8, 2006.

10: Akita Y, Matsumoto Y: Actual practice of topical ALA-PDT. MB Derma, 132: 142-149, 2007.

11: Braathen LR, Szeimies RM, Basset-Seguin N et al: International Society for Photodynamic Therapy in 
Dermatology. Guidelines on the use of photodynamic therapy for nonmelanoma skin cancer: and international consensus. International Society for Photodynamic Therapy in Dermatology, 2005. J Am Acad Dermatol, 56: 125-143, 2007.

12: Morton CA, McKenna KE and Rhodes LE: Guidelines for topical photodynamic therapy: update. Br J Dermatol, 159: 1245-1266, 2008.

13: Nakano A, Tamada Y, Watanabe D, et al: A pilot study to assess the efficacy of photodynamic therapy for Japanese patients with actinic keratoses in relation to lesion size and histological severity. Photoderm Photoimmunol Photomed, 25:37-40,
2009.

14: "Instructions for the use of laser devices", release no. 524 Notice from the Division Head of the Evaluation and Licensing Division, Pharmaceutical Affairs Bureau of the Japanese Ministry of Health, Labour and Welfare, dated April 22 ${ }^{\text {nd }}, 1980$.

15: Appendix 2 of "Rules and Regulations of the Manufacturer and Distributor" from the business communications of the Division of Medical Device Development, Pharmaceutical Affairs Bureau of the Japanese Ministry of Health, Labour and Welfare, dated August 6 ${ }^{\text {th }}, 1991$.

These guidelines were translated from

Safety Guidelines for Laser Surgery and Medicine - 2011 version, supplement of

The Journal of Japan Society for Laser Surgery and Medicine, Vol. 32,

with the permission of the Safety Committee of

the Japan Society for Laser Surgery and Medicine 